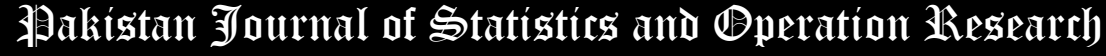

\section{Convergence Rates of Maxima of Non-identical Sums}

\author{
Ibrahim A. Ahmad ${ }^{1}$, Netti Herawati ${ }^{2 *}$
}

* Corresponding Author

1. Department of Statistics, Oklahoma State University, Stillwater, OK, USA, drabe_aa@yahoo.com

2. Department of Mathematics, University of Lampung, Bandar Lampung, Indonesia, netti.herawati@fmipa.unila.ac.id

\begin{abstract}
For a sequence of independent non-identically distributed random variables with positive means, rates of convergence of the maximum of their sums are established. These rates are exact and are obtained under the same moment conditions as those used for partial sums.
\end{abstract}

Key Words: Rates of convergence; maximum partial sums; central limit theorem

\section{Mathematical Subject Classification: 60F05}

\section{Introduction}

Let $\left\{X_{n}\right\}$ be a sequence of independent random variables not necessarily identically distributed such that $X_{n}$ has a distribution function $F_{n}$ with $E X_{n}=\mu_{n} \geq a>0$ and $V\left(X_{n}\right)=\sigma_{n}^{2}<\infty, n \geq 1$. Set $S_{n}=\sum_{i=1}^{n} X_{i}, A_{n}=\sum_{i=1}^{n} \mu_{i}$ and $V_{n}^{2}=\sum_{i=1}^{n} \sigma_{i}^{2}$. Also define $\beta_{n}^{2+\delta}=E\left|X_{n}-\mu_{n}\right|^{2+\delta}$ and set $B_{n}^{2+\delta}=\sum_{i=1}^{n} \beta_{i}^{2+\delta}$ for $0<\delta \leq 1$. Further define the sequence of maximum partial sums $S_{n}^{*}=\max _{1 \leq i \leq n} S_{i}$. Set

$$
\begin{aligned}
& \Delta_{n}=\sup _{x}\left|P\left(S_{n}-A_{n} \leq x V_{n}\right)-\Phi(x)\right|, n \geq 1, \\
& \Delta_{n}^{*}=\sup _{x}\left|P\left(S_{n}^{*}-A_{n} \leq x V_{n}\right)-\Phi(x)\right|, n \geq 1 .
\end{aligned}
$$

The rate at which $\Delta_{n}$ converges to zero was first considered by Berry (1941) and was followed independently by Esseen (1945). They showed that when the sample is identically distributed with $\delta=1$, then $\Delta_{n}=O\left(n^{-\frac{1}{2}}\right)$. Katz (1963) showed that for any $0<\delta \leq 1$, then $\Delta_{n}=O\left(n^{-\frac{\delta}{2}}\right)$.

On the other hand, Heyde (1967) had shown that $\sum_{n=1}^{\infty} n^{-1+\frac{\delta}{2}} \Delta_{n}<\infty$ for all $0<\delta<1$ and that when $\delta=0$ then $\sum_{n=1}^{\infty} n^{-1} \Delta_{n}<\infty$.

When the observation $X_{i}^{\prime}$ s are non-identically distributed Petrov (1995) showed that $\Delta_{n}=O\left(n^{-\frac{\delta}{2}}\right)$ for any $0<$ $\delta \leq 1$, while Serova (1979) showed that $\sum_{n=1}^{\infty} n^{-1+\frac{\delta}{2}} \Delta_{n}<\infty$, for all $0<\delta<1$ and when $\delta=0, \sum_{n=1}^{\infty} n^{-1} \Delta_{n}<$ $\infty$. For latest development see DasGupta (2008) and Senatov (1998).

As for $\Delta_{\mathrm{n}}^{*}$, Ahmad (1979) showed that in the identically distributed random variables $\Delta_{\mathrm{n}}^{*}=O\left(n^{-\frac{\delta}{2}}\right)$ when $0<\delta \leq$ 1 and that $\sum_{n=1}^{\infty} n^{-1+\frac{\delta}{2}} \Delta_{\mathrm{n}}^{*}<\infty$ for all $0<\delta<1$ and that $\sum_{n=1}^{\infty} n^{-1} \Delta_{\mathrm{n}}^{*}<\infty$. Hence we present analogous results for non-identical case. The results of $\Delta_{\mathrm{n}}^{*}$ presented here are the main tools in deriving the asymptotic normality rates of the first time passage of random variables. The first passage time is defined by: $U(t)=\inf \left\{n=\sum_{i=1}^{n} X_{i}>t\right\}$. 
Thus, $P[U(t) \leq m]=P[S * \geq t]$. Central limit theorem of $U(t)$ was given by Siegmund (1968) among others and its rate of convergence are being developed by the authors at the moment where we will use the results there in approximating the rate of convergence of $U(t)$.

\section{Rates of Convergence of $\Delta_{n}^{*}$}

The following two theorems give basic rate of convergence results for $\Delta_{n}^{*}$.

Theorem 2.1. Let $\beta_{n}^{2+\delta}=E\left|X_{n}-\mu_{n}\right|^{2+\delta} \leq M<\infty$ for some constant $M>0$ for all $n \geq 1$ and set $B_{n}^{2+\delta}=$ $\sum_{i=1}^{n} \beta_{i}^{2+\delta}$. Then

$$
\Delta_{n}^{*}=O\left(B_{n}^{2+\delta} / V_{n}^{2+\delta}\right), 0<\delta \leq 1 .
$$

Theorem 2.2. Let there exist a random variable $X$ such that $V(X)<\infty$ and let $\gamma>0$ and $x_{0}>0$ be constants for which the following condition hold,

$$
\sum_{i=1}^{n} P\left[\left|X_{i}\right| \geq x\right] \leq \gamma V_{n}^{2} P[|X| \geq x],
$$

for all $x>x_{0}$. Assume that $E X^{2} \ln (1+|X|)<\infty$ if $\delta=0$ and that $E|X|^{2+\delta}<\infty$ if $0<\delta<1$. Then

$$
\sum_{n=1}^{\infty} \frac{\sigma_{n}^{2}}{V_{n}^{2+\delta}} \Delta_{n}^{*}<\infty
$$

In what follows, $\mathrm{C}$ is used for positive generic constants not necessarily equal.

Proof of Theorem 2.1. It follows from a result of Petrov (1995) that under the assumption of the theorem $\Delta_{n} \leq$ $C B_{n}^{2+\delta} / V_{n}^{2+\delta}$ for all $n \geq 1$ and some $C>0$. Thus we need to establish that for all $x$,

$$
\gamma_{n}=P\left[S_{n} \leq x\right]-P\left[S_{n}^{*} \leq x\right]=O\left(B_{n}^{2+\delta} / V_{n}^{2+\delta}\right) .
$$

But

$$
\begin{aligned}
\gamma_{n} \leq & \max _{1 \leq i \leq[n / 2]} P\left[S_{n}-S_{i}<0\right] \sum_{i=0}^{[n / 2]} P\left[S_{0} \leq x, \ldots, S_{i-1} \leq x, S_{i}>x\right] \\
& \quad+\sum_{i=[n / 2]+1}^{n-1} P\left[S_{0} \leq x, \ldots, S_{i-1} \leq x, S_{i}>x\right] P\left[S_{n}-S_{i}<0\right] \\
= & I_{1 n}+I_{2 n}, \text { say, }
\end{aligned}
$$

where $[x]$ denotes the largest integer less than equal to $x$. Now,

$$
\begin{aligned}
I_{1 n} & \leq \max _{1 \leq i \leq[n / 2]} P\left[S_{n}-S_{i}<0\right]=\max _{1 \leq i \leq[n / 2]} P\left[S_{n}-S_{i}-\left(A_{n}-A_{i}\right)<-\left(A_{n}-A_{i}\right)\right] \\
& \leq\left[\max _{1 \leq i \leq[n / 2]} \frac{V\left(S_{n}-S_{i}\right)}{\left(A_{n}-A_{i}\right)^{2}} \frac{V_{n}^{2+\delta}}{B_{n}^{2+\delta}}\right]\left[\frac{B_{n}^{2+\delta}}{V_{n}^{2+\delta}}\right] \\
& =\left[\max _{1 \leq i \leq[n / 2]} \frac{\sum_{l=i+1}^{n} \sigma_{l}^{2}}{\left(\sum_{l=i+1}^{n} \mu_{l}\right)^{2}} \frac{V_{n}^{2+\delta}}{B_{n}^{2+\delta}}\right]\left[\frac{B_{n}^{2+\delta}}{V_{n}^{2+\delta}}\right] \leq\left[\max _{1 \leq i \leq[n / 2]} \frac{(n-i) M}{(n-i)^{2} a}\right]\left[\frac{B_{n}^{2+\delta}}{V_{n}^{2+\delta}}\right] \\
& \leq C n^{-1}\left[\frac{B_{n}^{2+\delta}}{\left.V_{n}^{2+\delta}\right] .}\right.
\end{aligned}
$$

Next,

$$
\begin{aligned}
I_{2 n} & \leq \max _{[n / 2]+1 \leq i \leq n-1} P\left[S_{n-1}^{*} \leq x, S_{n}>x\right] \sum_{i=[n / 2]+1}^{n-1} P\left[S_{n}-S_{i}<0\right] \\
& \leq \max _{[n / 2]+1 \leq i \leq n-1} P\left[S_{i-1} \leq x, S_{i}>x\right] \sum_{i=[n / 2]+1}^{n-1} P\left[S_{n}-S_{i}<0\right] \\
& =J_{1 n} . J_{2 n}, \text { say. }
\end{aligned}
$$


But

$$
\begin{aligned}
J_{1 n} & =\max _{[n / 2]+1 \leq i \leq n-1} \int_{0}^{\infty}\left\{P\left[S_{i-1} \leq x\right]-B\left[S_{i-1} \leq x-y\right]\right\} d F_{i}(y) \\
& \leq \max _{[n / 2]+1 \leq i \leq n-1}\left\{2 C\left(\frac{B_{i-1}^{2+\delta}}{V_{i-1}^{2+\delta}}\right)+\int_{0}^{\infty}\left|\Phi\left(\frac{x-A_{i-1}}{V_{i-1}}\right)-\Phi\left(\frac{x-y-A_{i-1}}{V_{i-1}}\right)\right| d F_{i}(y)\right\} \\
& \leq \max _{[n / 2]+1 \leq i \leq n-1}\left\{2 C\left(\frac{B_{i-1}^{2+\delta}}{V_{i-1}^{2+\delta}}\right)+\frac{\beta_{i}^{1 / 3}+C}{\sqrt{2 \pi} V_{i-1}}\right\} \\
& \leq \max _{[n / 2]+1 \leq i \leq n-1}\left\{C\left(\frac{B_{i-1}^{2+\delta}}{V_{i-1}^{2+\delta}}\right) \leq C \frac{B_{[n / 2]}^{2+\delta}}{\left.V_{[n / 2]}^{2+\delta}\right\}},\right.
\end{aligned}
$$

since $|\Phi(x)-\Phi(x+\epsilon)| \leq \frac{|\epsilon|}{\sqrt{2 \pi}}$ and $\frac{B_{n}^{2+\delta}}{V_{n}^{2+\delta}}$ will eventually decrease to zero. Next,

$$
J_{2 n} \leq \sum_{i=[n / 2]+1}^{n-1} P\left[S_{n}-S_{i} \leq 0\right] .
$$

But using the theorem of Petrov (1995), cf (by Ahmad \& Lin, 1977), we get:

$$
\begin{aligned}
P\left[S_{n}-S_{i}<0\right]= & \Phi\left(-\frac{A_{n}-A_{i}}{\left(\sum_{l=i+1}^{n} \sigma_{l}^{2}\right)^{1 / 2}}\right)+C \frac{\sum_{l=i+1}^{n} \beta_{l}^{2+\delta}}{\left(\sum_{l=i+1}^{n} \sigma_{l}^{2}\right)^{1+\delta / 2}} \cdot \frac{\left(\sum_{l=i+1}^{n} \sigma_{l}^{2}\right)^{1+\delta / 2}}{\left(\sum_{l=i+1}^{n} \mu_{l}\right)^{2+\delta}} \\
& \leq C\left\{\frac{\left(\sum_{l=i+1}^{n} \sigma_{l}^{2}\right)^{3 / 2}}{\left(\sum_{l=i+1}^{n} \mu_{2}\right)^{3}}+\frac{\sum_{l=i+1}^{n} \beta_{l}^{2+\delta}}{\left(\sum_{l=i+1}^{n} \mu_{l}\right)^{2+\delta}}\right\} \\
& \leq C\left\{\frac{\left(\sum_{l=i+1}^{n}\left(\beta_{l}^{2+\delta}\right)^{2 /(2+\delta)}\right)^{3 / 2}}{\left(\sum_{l=i+1}^{n} \mu_{l}\right)^{3}}+\frac{\sum_{l=i+1}^{n} \beta_{l}^{2+\delta}}{\left(\sum_{l=i+1}^{n} \mu_{l}\right)^{2+\delta}}\right\} \\
& \leq C\left\{(n-i)^{-3 / 2}+(n-i)^{-1-\delta}\right\},
\end{aligned}
$$

where in the first inequality we used the fact that for any $x>0, \Phi(-x)=O\left(x^{-3}\right)$, and in the second inequality, we used the facts that $\mu_{n} \geq a, \sigma_{n}^{2} \leq\left(\beta_{n}^{2+\delta}\right)^{2 /(2+\delta)}$, and $\beta_{n} \leq M$ for all $n$. Hence

$$
\sum_{i=[n / 2]+1}^{n-1} P\left[S_{n}-S_{i}<0\right] \leq C\left\{\sum_{i=[n / 2]+1}^{\infty}(n-i)^{-\frac{3}{2}}+\sum_{i=[n / 2]+1}^{\infty}(n-i)^{-1-\delta}\right\} \leq C .
$$

Hence $I_{2 n}=O\left(B_{n}^{2+\delta} / V_{n}^{2+\delta}\right)$ as $n \rightarrow \infty$ and the theorem is now proved. ॥

Proof of Theorem 2.2. The structure of the proof is two stages. First we show that $\sum_{n=2}^{\infty} \frac{\sigma_{n}^{2}}{V_{n}^{2-\delta}} \Delta_{n}<\infty$ and then we show that for any real $x, \sum_{n=2}^{\infty} \frac{\sigma_{n}^{2}}{V_{n}^{2-\delta}}\left\{P\left[S_{n} \leq x\right]-P\left[S_{n}^{*} \leq x\right]\right\}<\infty$. The first assertion follows from the work of Serova (1979). Thus we need to establish the second assertion. Recall that in (2.5) we have $I_{1 n} \leq C n^{-1}\left[\frac{B_{n}^{2+\delta}}{V_{n}^{2+\delta}}\right]$, then we have

$$
\sum_{n=2}^{\infty} \frac{\sigma_{n}^{2}}{V_{n}^{2+\delta}} I_{1 n} \leq C \sum_{n=2}^{\infty} \frac{\sigma_{n}^{2} B_{n}^{2+\delta}}{n V_{n}^{4}}<\infty,
$$

where the convergence is assured since this behaves like $\sum n^{-2+\delta}<\infty$. Next it is sufficient to show that $\sum_{n=2}^{\infty} \frac{\sigma_{n}^{2}}{V_{n}^{2-\delta}} I_{2 n}<\infty$. But as in Theorem 2.1 we can easily see that: 


$$
\begin{aligned}
\sum_{n=1}^{\infty} \frac{\sigma_{n}^{2}}{V_{n}^{2-\delta}} I_{2 n} \leq & C \sum_{n=2}^{\infty} \frac{\sigma_{n}^{2}}{V_{n}^{2-\delta}} \max _{[n / 2]+1 \leq i \leq n-1} \int\left\{\left|P\left[\frac{S_{i-1}-A_{i-1}}{V_{i-1}} \leq \frac{x-A_{i-1}}{V_{i-1}}\right]-\Phi\left(\frac{x-A_{i-1}}{V_{i-1}}\right)\right|\right. \\
& +\left|P\left[\frac{S_{i-1}-A_{i-1}}{V_{i-1}} \leq \frac{x-y-A_{i-1}}{V_{i-1}}\right]-\Phi\left(\frac{x-y-A_{i-1}}{V_{i-1}}\right)\right| \\
& \left.+\left|\Phi\left(\frac{x-A_{i-1}}{V_{i-1}}\right)-\Phi\left(\frac{x-y-A_{i-1}}{V_{i-1}}\right)\right|\right\} d F_{i}(x) \\
\leq & C\left(J_{1}+J_{2}+J_{3}\right), \text { say. }
\end{aligned}
$$

Let us evaluate each term separately,

$$
\begin{aligned}
& \quad J_{1} \leq \sum_{n=2}^{\infty} \frac{\sigma_{n}^{2}}{V_{n}^{2-\delta}} \max _{[n / 2]+1 \leq i \leq n-1} \int_{-\infty}^{\infty}\left|P\left[\frac{S_{i-1}-A_{i-1}}{V_{i-1}} \leq \frac{x-A_{i-1}}{V_{i-1}}\right]-\Phi\left(\frac{x-A_{i-1}}{V_{i-1}}\right)\right| d F_{i}(x) \\
& \leq \sum_{n=2}^{\infty} \frac{\sigma_{n}^{2}}{V_{n}^{2-\delta}} \max _{[n / 2]+1 \leq i \leq n-1} \Delta_{i} \leq \sum_{n=2}^{N_{0}} \frac{\sigma_{n}^{2}}{V_{n}^{2-\delta}} \max _{[n / 2]+1 \leq i \leq n-1} \Delta_{i}+\sum_{n=N_{0}+1}^{\infty} \frac{\sigma_{n}^{2}}{V_{n}^{2-\delta}} \Delta_{[n / 2]+1} \\
& \leq L_{1 n}+\sum_{n=2}^{\infty} \frac{\sigma_{n}^{2}}{V_{n}^{2-\delta}} \Delta_{n}<\infty,
\end{aligned}
$$

since for $n<N_{0}, \Delta_{n}$ decrease to 0 . Similarly we can show that $J_{2}<\infty$. Finally,

$$
\begin{aligned}
& J_{3} \leq C \sum_{n=2}^{\infty} \frac{\sigma_{n}^{2}}{V_{n}^{2-\delta}} \max _{[n / 2]+1 \leq i \leq n-1} \frac{1}{V_{i}-1} \int_{-\infty}^{\infty}|y| d F_{i}(y) \\
& \leq C \sum_{n=2}^{\infty} \frac{\sigma_{n}^{2}}{V_{n}^{3-\delta}}<\infty .
\end{aligned}
$$

Hence the second assertion is proved and so is the theorem. ॥

\section{References}

1. Ahmad, I.A. \& Lin, P.E. (1977). A Berry-Essen type theorem. Utilitas Math., 11, 153-160.

2. Ahmad, I.A. (1979). A note on rates of convergence in the multidimensional CLT for maximum partial sums. J. Mult. Analysis, 9, 314-322.

3. Berry, A.C. (1941). The accuracy of the Gaussian approximation to the sum of independent variables. Trans. Amer. Math. Soc., 49, 122-136.

4. DasGupta, A. (2008). Asymptotic theory of statistics and probability. Springer.

5. Esseen, C.G. (1945). Fourier analysis of distribution functions. A mathematical study of the LaplaceGaussian Law. Acta. Math., 77, 1-125.

6. Katz, M. (1963). Note on the Berry-Esseen theorem. Ann. Math. Statist., 34, 1107-1108.

7. Heyde, C.C. (1967). On the influence of moments on the rate of convergence to the normal distribution. Z. Wahrscheinlichkeitstheorie verw. Geb., 8, 12-18.

8. Petrov, V.V. (1995). Limit Theorems of Probability Theory. Oxford University Press, Oxford.

9. Serova, G.V. (1979). On the rate of convergence in the central limit theorem. Theor. Prob. Math. Statist., 17, 121-130.

10. Siegmund, D. (1968). On the asymptotic normality of one-sided stopping rules. Ann. Math. Statist., 39, 1493-1497.

11. Senatov, V.V. (1998). Normal approximation: New results, methods and problems. VSP, Utrecht. 